Miša Glišič

Študentka Oddelka za germanistiko

Filozofska fakulteta Univerze v Ljubljani

glisic.misa@gmail.com
UDK 821.112.2(436).09-32Handke P.

DOI: $10.4312 /$ vestnik.8.169-185

\title{
DIE KONSTRUKTION DES KULTURELL FREMDEN IN PETER HANDKES DIE MORAWISCHE NACHT
}

\section{EINLEITUNG}

Peter Handkes Erzählung Die Morawische Nacht ist eine romanlange „Unterwegserzählung" eines namenlosen Ex-Autors über sein Leben, seine Wünsche, Träume und Fantasien. Handke erschafft mit der literarischen Figur des Autors ein anderes Ich, welches über seine Verlorenheit der eigenen Seele auktorial ${ }^{1}$ erzählt und sich auf die Suche nach seiner eigenen Identität im plurikulturellen Raum des Balkans begibt. Der Ausgangspunkt der Erzählung ist ein fiktiver Ort am Fluss Morawa. Weitere Orte, die im Buch vorkommen, sind: Belgrad, Krk, das kastilische Hochland in Spanien, der Harz in Deutschland, Wien, Gutenstein am Fuße des Semmering, Graz, Triester Karst und schließlich auch Kärnten als der verlorene Heimatort des Autors. Das literarische Meisterwerk eröffnet mit seiner poetischen Sprache und seinem Esprit anhand verschiedener Orte und Kulturen, die in der Erzählung auftreten, einen interkulturellen Dialog. Die ineinander verflochtenen Geschichten der literarischen Figur des Schriftstellers bzw. „Ex-Autors“ weisen auch autobiografische Züge von Handke auf. Der Autor schreibt sich in dem sozialen Raum ein und tritt als Beobachter auf. Die Erzählinstanz, die mit dem Protagonisten der Erzählung gleichzusetzen ist, fühlt sich in seiner eigenen Zeit fremd und versucht in einer imaginären Ordnung heimisch zu werden. Das Individuum strebt nach Freiheit und Ruhe und nimmt die Umgebung mit einem distanzierten Blick des Anderen wahr. Aus der Beobachterperspektive der zentralen literarischen Figur werden verschiedene Weisen des Fremdverstehens beschrieben. Der Umgang mit dem Fremden erhält eine ethnische und politische Dimension. Durch kulturreflexives Erzählen versucht Handke eine Sensibilität für kulturelle Differenzbestimmungen zu erzeugen. Die Fremdheit als ästhetisches Verfahren in dem literarischen Werk konstruiert eine innere Geschichte eines zerspaltenen

1 Anhand einer distanzierten auktorialen Perspektive kann keine Realitätsbindung entstehen, sondern eine Verfremdung von Wahrnehmungskategorien. Der Wechsel von Erzählperspektiven schafft Distanz zum Erzählten und einen Identitätsschutz zugleich. Die Erzählstrategie mit Verwendung verschiedener Erzählinstanzen und Personalpronomina markiert differente Positionen, die als Eigenheit und Fremdheit aufgefasst werden können. Diese setzen eine relative Indifferenz, die soziale Verhältnisse als gleichgültig erscheinen ließe, voraus (vgl. Waldenfels 2013: 74). 
Ich, bei dem eine autobiografische Subjektzentrierung präsent ist. Der Protagonist der Erzählung, genauer gesagt der Autor, verkörpert die Hybridität, indem er verschiedene Länder besucht und sich zwischen den Kulturen bewegt. Als Reisender versucht er seine Zugehörigkeit kreativ zu entfalten. Die Alteritäts- und Identitätsmomente werden anhand des Diskurses innerhalb der Erzählung veranschaulicht. Es stellt sich die Frage nach der leiblichen Welterschließung und Neuverortung von Identitäts- und Repräsentationsfragen in dem Untersuchungsfeld von kultureller Differenz, Alterität und Macht, mit dem sich der Beitrag im Weiteren befassen wird.

\section{2 INTERKULTURALITÄT ALS PRINZIP DES KULTURELL ANDEREN UND FREMDEN}

Die Interkulturalität schafft Übergänge und Zwischenräume im Rahmen eines Spannungsfeldes, das Möglichkeiten eröffnet (vgl. Wierlacher 1985, zit. nach Wierlacher 2003: 259). Handke konstruiert das kulturelle Feld aus der Perspektive seiner eigenen Erfahrung als Diskursteilnehmer und Protagonist. Er setzt sich mit kulturellen Differenzen und Pluralität auseinander. Die eigenen Werte, Normen und Gewohnheiten werden durch die Konfrontation mit der Fremdheit provoziert. Der Protagonist versucht seine eigene Identität durch das „Andere“ zu konstituieren. Die Überlagerung verschiedener Zugehörigkeiten und Lebenswelten sorgen für einen Zwiespalt der literarischen Hauptfigur, die durch hybride ${ }^{2}$ Situationen geprägt wird. Der „Ex-Autor“ bewegt sich in einem sogenannten Zwischenraum, der nach Bhabhas postkolonialer Theorie (Bhabha 2000) als „Dritter Raum“3 angesehen werden kann: „Zwischenwässern “: hieß so nicht auch sein, des Erzählers der Morawischen Nacht, Herkunftsort? Wie es ihn, den Feuermenschen, doch seit jeher ans Wasser zog, ans fließende " (MN, 259) ${ }^{4}$.

„Dritter Raum' erweist sich als eine spezifische Existenzform der Selbstverfremdung durch Migration, zugleich aber auch als Ausgangspunkt eines kulturwissenschaftlichen Konzepts von transnationaler ,hybrider' Kulturüberlagerung“" (Bachmann-Medick 1999: 521). In diesem Raum wird das Dasein der literarischen Figur erst ermöglicht. Ein solcher Raum tritt in dem literarischen Text in Form eines fiktiven Schiffes ,zwischen dem Dorf Porodin und der Stadt Velika Plana “ (MN, 8) mit dem Namen Morawische Nacht auf. Das Schiff hat „die Funktion eines intermediären Feldes, in dem Fremdheitserfahrungen in der kontrastiven Profilierung unterschiedlicher kultureller Erfahrungshorizonte imaginär inszeniert werden“" (vgl. Gutjahr 2002: 363). Davon zeugt auch folgendes Zitat aus dem Buch:

2 Die Hybridität zeigt sich auch als innere Differenzierung einer inneren Kultur, die von dem Subjekt geprägt wird.

3 Im Sinne eines Ortes, an dem reale und imaginäre Örtlichkeiten zugleich präsent sind. Es handelt sich dabei um einen Ort der Auseinandersetzung in und zwischen den Kulturen.

4 Peter, Handke: Die Morawische Nacht. Erzählung, Frankfurt am Main: Suhrkamp Taschenbuch Verlag 2009. Im Folgenden MN und Seitenangabe. 
Warum hatte unser Bootsmann gerade die Gegend von Porodin zu seinem Wohnort gemacht? Wir konnten nur rätseln: Die einen meinten, das komme von der balkanweit verbreiteten Geschichte zwischen den Kriegen - es war da immer, wenn nicht Krieg, so „zwischen den Kriegen“ gewesen -, wonach in dem Gemeindegebieten ein Haustier von einem Einheimischen ermordet wurde, worauf das ganze Dorf dafür an jedem Jahrestag Sühne leistete. (MN, 9f.)

Ein solches Kulturkonzept bringt auch Verschwiegenes und Unbewusstes innerhalb von Kulturen ans Licht. Mit Hilfe des Zwischenraumes macht sich der Autor ein Bildnis von seiner eigenen Persönlichkeit. In diesem Zwischenraum entwickelt sich die primäre Geschichte der Erzählung. Freunde werden von dem Autor auf sein Schiff eingeladen und persönliche Geschichten des Autors werden in Form von Binnenerzählungen in einer Nacht auf der Morawa erzählt. Daraus erfolgt eine Vielfalt von Geschichten und Gedanken, die für eine mysteriöse Atmosphäre sorgt. Das Erzählen ermöglich einen distanzierten und fremden Einblick in die kulturelle Realität. Nach dem Theoriekonzept der Alterität und Interkulturalität von Ortrud Gutjahr (2002) entstehe die Interkulturalität über die Konstruktionsprozesse von Differenz und das Festlegen von Identität und Alterität. Alois Wierlacher (2003: 257) definiert den Begriff der Interkulturalität folgendermaßen: „Der Begriff der Interkulturalität wird auch als Bezeichnung eines auf Verständigung gerichteten realen oder dargestellten menschlichen Verhaltens in Begegnungssituationen verstanden, an denen einzelne Menschen oder Gruppen aus verschiedenen Kulturen in diversen zeitlichen continua beteiligt sind." Der Autor führt hybride ${ }^{5}$ Situationen vor und lässt sie auf sich als Beobachter einwirken. So fragt er in seiner Erzählung:

Dann einmal lag der Balkan hinter ihm. Erleichterung. Was für eine Erleichterung. Aber woran zeigte sich, daß man heraus aus dem Balkan war? An der Meeresluft, an den ersten Büscheln des wilden Thymian, Rosmarin, an einem Lorbeerstrauch, einer ersten, noch einzelnen Palme, so wie es etwa Ivo Andrić von seinen Jugendfahrten aus den Kesseltälern des Landesinnern, weg von der gebirgskalten Drina an die Adria, in seiner Sprache den Jadran erzählt hat? (MN, 121)

Die Verflechtung der Kulturen kommt bei der geographischen Räumlichkeit, wo verschiedene Regionen aufeinandertreffen und eine sog. ,Transregionalität“ bilden, zum Ausdruck. Aus den rhetorischen Fragen im Zitat wird deutlich, dass die Grenzen zwischen den Kulturen nicht deutlich gezogen werden können, denn Kulturen beeinflussen sich gegenseitig. Die nichthomogenen Konstellationen innerhalb der Kulturen führen zu transkulturellen Interaktionen zwischen den Kulturen und wechselseitigem Ineinanderwirken. Hier stellt sich vor allem die Frage, wo die jeweilige Kultur überhaupt zu verorten ist?

5 Hybridität als postkolonialer Leitbegriff bezeichnet die Fruchtbarkeit kultureller Vermischungen jenseits kultureller Reinheit (vgl. Bachmann-Medick 2006: 197). 
Bei dem Protagonisten ruft die Insel Krk ein abstoßendes Gefühl hervor: „Bei allem Hochbetrieb wehte ihn von der Insel seines ersten Buchs eine leere Welt an, und diese Leere, statt ihn, wie sonst oft, willkommen zu heißen, stieß ihn zurück; leistete ihm Widerstand" (MN, 127). Die Umgebung hat bei dem Integrationsversuch einen negativen Einfluss auf den Ex-Autor. Er kommt auch mit sprachlichen Differenzen, die für ein Unverständnis in der interkulturellen Kommunikation sorgen, nicht zurecht:

Als er damals nach dem Schreiben weit hinaus ins Meer geschwommen war, hatten ihn vom Ufer aus Rufe wie „Meerhund! Meerhund!“ erreicht, wobei er sich nichts gedacht hatte und einfach weitergeschwommen war, und erst später, beim Nachschauen im Wörterbuch, sollte er lesen, daß „Meerhund“ der Landesname für „Hai“ war. (MN, 128)

Das oben genannte Beispiel verweist auf eine falsche kulturelle De-Codierung der Mitteilung. Die unverständliche fremde Sprache der Landschaft führt zur einer Fremderfahrung des Protagonisten und verstärkt das Entfremdetsein. Die Fremderfahrung entsteht durch Ausgrenzung und ruft eine Ferne in der Nähe, eine Abwesenheit in der Anwesenheit hervor (vgl. Waldenfels 2013: 146).

\section{3}

EIGEN- UND FREMDKULTURELLES BEZUGSFELD

Eigenes entsteht nicht durch Kontrastierung, denn Eigenes und Fremdes verflechten und verschränken sich miteinander (vgl. Waldenfels 2013: 156f.). Die kulturelle Fremdheit kann demnach nur als relationale Kategorie verstanden werden, denn das Eigene und das Fremde ergänzen sich in ihrer Beziehung. Der Protagonist wird sich seiner eigenen Herkunft auf eine distanzierte und fremde Weise bewusst:

„Warum scheue ich, seit meiner Herkunft hier [...], so zurück vor dem direkten Weg heimzu, ins Dorf, von wo ich herkomme, oder was davon übrig ist? Warum beschreibe ich Umweg um Umweg, Abstecher um Abstecher, um den Eintritt in mein Geburtshaus hinauszuschieben? Warum kommt mir vor, ich würde mich dort nähern einer verbotenen Zone? Einer Todeszone? Achtung, Todeszone!?“" (MN, 369f.)

„Das Fremde als das durch den Menschen prinzipiell Unerkennbare und Unfassbare nimmt die Ordnungsfunktion einer transzendenten Ganzheit ein, bei der es als tragender Grund und Resonanzboden von Eigenheit gedacht ist" (Gutjahr 2002: 360). Der Ex-Autor nimmt die bekannte Umgebung mit fremden Augen wahr und versucht anhand von Erinnerungen das Eigene in sich selbst zu finden: 
Und zugleich erkannte er den Baum in der Dorfmitte, den wohl abgestorbenen Kirschbaum, samt dem roh betonierten Quellhäuschen an seinem Fuß, und daneben das aufgelassene, immer noch gelbleuchtende Dorfwirtshaus, und gegenüber die Mauer rund um den fremden Obstgarten, über die man nicht nur einmal geklettert war zum Apfel- und Birnenstehlen. (MN, 402f.)

Ein weiteres Beispiel für die Auseinandersetzung mit dem Fremden wäre die Überzeugung des Protagonisten über die Sprachauswahl der Population: „Und es fiel ihm dann auf, daß er erwartet hatte, diese Leute würden eine andere Sprache sprechen, nicht deutsch, und wenn deutsch, so ein fremdes, ein ungewohntes altertümliches, eine Art Wolga-, Amazonas-, Mississippi, Yukon-, oder, warum nicht, Kongo-Deutsch“( (MN, 328).

\section{4}

\section{KULTUR ALS LITERARISCHE DARSTELLUNG}

Literatur als Erfahrungsraum kann aufgrund systemischer Eigenschaften als das Fremde an sich definiert werden und aufgrund der poetischen Sprache befremdend wirken bzw. Fremdheit erzeugen (vgl. Leskovec 2011: 61). Handkes kulturreflexives Erzählen spielt mit vertrauten Wahrnehmungsmustern und exponiert sie als fremd gewordene. Die Erzählung deckt auch postkoloniale Konstellationen innerhalb Europas auf. Kulturen werden in dem literarischen Werk dekonstruiert um die widersprüchlichen und mehrschichtigen Komponenten in den Kulturen zu veranschaulichen und für einen Diskurs zu öffnen. Der Protagonist ist mit einigen Passagieren im Bus unterwegs zu einem zerstörten Friedhof im geographischen Gebiet des Kosovo, wo sie gedenken wollen ihrer Vertriebenen und ihrer Toten. Der Konflikt zwischen der serbischen und albanischen Population wird schon bei der Busfahrt deutlich:

Zunächst wußte er, der erzählte, gar nicht, daß es ein Stein war, der jäh gegen das Fenster prallte. Es hörte und fühlte sich an wie ein heftiger Faustschlag, der nicht nur das Fenster traf, sondern den ganzen Bus. Die Passagiere aber reagierten nicht, auch er nicht. Das Glas, ein spezielles, war nicht zersplittert, bloß eingedellt, mit feinen Rissen, die ausstrahlten von der Delle. Erstmals dann eine Frage: „War das ein Stein?“‘, und als Antwort ein Nicken, ein kurzes; für die andern schien der Vorfall nichts Ungewohntes. (MN, 96f.)

Das wechselseitige Ineinanderwirken verschiedener, auch antagonistischer Kulturen wird in der Erzählung betont. Der Busfahrer beschreibt die präsente Situation dem ExAutor als Außenseiter folgenderweise:

Sie sind ein Staatsvolk und, o endlich wahrgemachter großer Traum, ein Einvolkstaat und hassen uns Überbleibsel vom Zweitvolk, das kein Staatsvolk 
ist, hassen uns, als seien wir Reste das Staatsvolk, und nicht sie. Und ihren Haß, den brauchen sie ihren Kindern gar nicht erst ausdrücklich beizubringen. Er überträgt sich einfach so, von Generation zu Generation, von Gen zu Gen, längst jenseits der Blutrachen und Kriege. [...] Euer Staat, er dient euch nur dazu, euern $\mathrm{Ha}$ auszuleben, im Schutz eurer Staatsgrenzen, Flaggen, die Drohflagen sind, und Hymnen, die Haßhymnen sind. Euern Haß auf jeden, der nicht eurer Staatsangehöriger ist, auf alles, was nicht Staat ist. (MN, 103f.)

Literatur ist Ausdruck der Kultur, denn die poetische Sprache eröffnet einen Zwischenraum, in dem auch die verdrängten und vergessenen Anteile der Kultur mit aufgehoben sind (vgl. Gutjahr 2002: 365). Handke äußerte sich über den realitätsbezogenen Vorfall im Bus auch persönlich mit folgenden Worten:

„Diese Völker haben nichts miteinander zu tun und dass sie sogar, in meiner Erfahrung - dass die kleinen Kinder, die zwei Jahre alt sind, automatisch Steine schmeißen, wenn sie einen Bus in kyrillischer, dass heißt in diesem Sinn der serbischen Aufschrift sehen - wo sind die Eltern, die Großeltern, die diesen lieben armen kleinen Kindern mal beibringen, dass ein Staat anders gegründet zu werden hat als aus einem ererbten Mechanismus der Gewalt?“ (ZDF 2008) ${ }^{6}$

Handke übernimmt die Rolle des Fragenden und des Zuhörers und des In-FrageStellers (vgl. Deichmann 2009: 190). „Die Differenz von Jenseits einer Grenze, von Drinnen und Draußen eines Bereichs läßt sich streng genommen nicht denken, ohne dass sich ein Eigenort von einem Fremdort abhebt, und sei es der operationale Eigenort dessen, der räumliche Vorgänge beobachtet und mißt" (Waldenfels 2013: 199f.). Peter Handke meint: „Man kann keinen Staat gründen auf Kosten eines Volkes, das in diesem Land, das dann Staat wird, lange gelebt hat, und das dieses Land als sein eigenes Land sieht “"(ZDF 2008).

\section{5}

\section{SELBSTFINDUNG DURCH FREMDERFAHRUNG}

Der Protagonist ist nicht sesshaft und repräsentiert die Funktion eines Heimkehrers, der sich mit der Transferierung des Unbekannten in das Vertraute beschäftigt. Unsere Hauptfigur lernt das Fremde kennen, indem sie sich räumlich bewegt und dabei Erfahrungen macht:

Nicht in dem Emigrantenbus wünschte er sich, aber doch weg von diesem Balkan, dem Balkan der Grenzstädte ohne dingfeste Grenzen, dem Balkan der tausend

6 Text und Bühne (2013): „Peter Handke im ZDF Nachtstudio Gespräch mit Volker Panzer am 10.3.2008“. [Youtube-Video] Verfügbar unter: https://www.youtube.com/watch?v=y8PM4YMEXhQ [zuletzt abgerufen am 2. September 2016]. Im Folgenden ZDF und Jahr der ersten Ausstrahlung. 
unsichtbaren, allesamt bösen und bitterfeindlichen Grenzen von Tal zu Tal, von Dorf zu Dorf, von Bach zu Bach, von Misthaufen zu Misthaufen, dem Balkan der steinschleudernden Kleinkinder, der menschenverachtenden Kußhände, des Knoblauchs, der die Vampire nur noch blutdürstiger machte. (MN, 113f.)

Die vertrauten Orientierungen und Denkmuster verlieren beim Raumtypus Fremde als das unbekannte Draußen ihre Gültigkeit (vgl. Gutjahr 2002: 360). Bhabha (2012: 36) vertritt den Standpunkt der politischen Theoretikerin Hannah Arendt, dass es ,[i]n einem integrierten Weltsystem von Nationalstaaten keinen ,Freiraum' - keinen eigenen Handlungs- und Meinungsspielraum - für die Staatenlosen, Flüchtlinge, Minderheiten, Vertriebenen oder apatrides [gäbe].“ In diesem Zusammenhang spricht auch Handke darüber, dass in der modernen Zeit keiner dieser Räume hinter den Grenzen existieren kann:

„Die Grenzen in Europa sind viel stärker und sogar kälter und böser geworden als sie von jeher waren. Die Grenzen, die es vorher gegeben hat, zwischen den Staaten, waren manchmal Anreize, zumindest für mich, ein Anreiz des Fremden. Ich habe das Fremde geträumt hinter den Grenzen und jetzt gibt es nichts Fremdes mehr zu träumen [...].“ (ZDF 2008)

Der Protagonist bekennt sich langsam wieder als Autor und möchte das Dasein auf einer ästhetischen Weise zu spüren bekommen um sich wiederzufinden. Er möchte von der Gesellschaft anerkannt werden und nicht mehr entfremdet sein: „Gesichter! Ein Gesicht! Gib mir ein Menschengesicht, und so wird meine Seele gesund" (MN, 378). Er findet zu sich selbst mit Hilfe einer begeisterten Leserin, die seine Aufmerksamkeit in einem Zug in Österreich weckt:

So einen Leser, so eine Leserin, so jemand durch und durch Zarten, hatte er sich vorzeiten auch für seine Bücher vorgestellt - so ein Wesen; an solcherart Lesen hätte er die erkannt, auf der Stelle, „mit hundertprozentiger Sicherheit“. Selbst wenn das gelesene Buch nicht von ihm gewesen wäre: er hätte sein Verfasser sein können, gemessen allein schon am Lidschlag des/der Lesenden, ein Lidschlag befreit von sämtlichen Automatismen, ein Lidschlag, welcher ein bewußtes Innehalten anzeigte - Bewußtwerden als ein Innehalten. Geduld ging über von ,seiner“ Leserin auf ihn; und in der Folge die umfassende Vorstellung: ihr Lesen sei ein Beschützen; indem sie so las, wie sie las, half sie jemandem, der in Gefahr war. Schutz gebend wirkte so ein Lesen, Geleit gebend, bewahrend. [...] „Ja“, sagte er dazu wörtlich auf dem Schiff: „Ich erachte meine Leser höher als mich selber.“ (MN, 382f.) 


\section{6 \\ EXISTENZ- UND IDENTITÄTSFRAGE}

Handke verhandelt seine kulturelle Identität in einer diskontinuierlichen und intertextuellen Zeitlichkeit der kulturellen Differenz. „Die kulturelle und politische Identität werden erst durch einen Prozess der Alterisierung herausgebildet, durch Einschalten einer Alteritätsperspektive, die das Selbst verfremdet und als Anderes erkennt" (Bachmann-Medick 2006: 206). Bei dem Autor ist ein ambivalentes Verhältnis zu seiner Berufsorientierung zu erkennen: ,Außerdem sagte er sich, daß die Gefahr, wenn nicht von außen, so verläßlich aus ihm selber käme. Er persönlich verkörperte die Gefahr, war seine Bedrohung, insbesondere seit er seinen Beruf hatte sein lassen - nicht mehr arbeitete" (MN, 375).

Der Protagonist findet sich selbst durch die Fremderfahrung. Wahrnehmungen, Emotionen, Sprachen und Bewusstseinsprozesse haben eine entscheidende Rolle bei der Konstruktion der Identität des Subjekts. Der Protagonist wird mit seiner kulturellen Bezugnahme zum Fremden und Unverstehbaren. Die Hauptfigur ist in ihrer Selbst- und Fremdwahrnehmung gespalten: „Und ob es einzig zum Scherz war, wenn er ständig wiederholte, er habe das Schreiben sein lassen auch aus zunehmendem Widerwillen gegen die Geräusche, gleichwelche? Ein jedes Geräusch habe er mit der Zeit als Krach empfunden, als Lärm, bösartigen " (MN, 16).

Emotionalität und Empathie bilden einen Teil der Persönlichkeit des Protagonisten, die am deutlichsten bei der Erzählung der Geschichte über die Busfahrt zum Ausdruck kommt:

Und zugleich, ja, zugleich ging dieses Herz, dasselbe Herz, ihm auf, nahm Gestalt an, und blutete, wie es, so kam ihm vor, seit „einer ganzen Ewigkeit“ nicht mehr geblutet hatte. Nicht eingezwängt in die Menge da fühlte er sich, sondern frei von ihr, frei durch sie, so frei, wie er in allen den Jahren [...] nie gewesen war. (MN 54)

Handke beweist in seiner Erzählung, dass für das kulturelle Verständnis und Selbstverständnis die biologische Identität und ethnische Herkunft nicht mehr entscheidend sind. Anhand des Protagonisten kann die Auffassung vertreten werden, dass nicht die Identität, sondern die Identifikation des Menschen wichtig ist. Der Begriff der Identität wird der Essenz entfremdet. Die Identifikation ist ein Symbol der eigenen Selbsterkenntnis und sie bildet somit den Höhepunkt der eigenen Existenz. Anhand der Identifikation projiziert sich das Individuum in der Gesellschaft und erschafft eine Ich-Konstellation, mit der es nicht manipuliert werden kann. Alles andere wäre eine Dekonstruktion des eigenen Ich, was auch die Erzählinstanz feststellt:

„Ja, verdammter Vaterloser du! [...] Ein Verbannter bist du, verbannt nicht allein aus der Vaterstadt, sondern von allen Stätten, ein Ortloser bist du. Den Prinz gabst, Vaterloser, du, mit freiem Raum um dich, und warst, wenn Prinz, 
doch nur Prinz von Nirgendwo, Prinz-ohne-Raum. [...] Wirst nie zur Menschheit gehören. [...] Weg mit dir, mein Lieber. Du hast hier nichts zu suchen. Srečan put. Gute Weiterreise.“ (MN, 303ff.)

Die Existenz der literarischen Hauptfigur ist auch an die Natur gebunden, denn der Autor bekennt sich schon sein ganzes Leben lang auch als ein „Obstgartendieb“:

Das Obstdiebstum machte einen Teil seines Selbstbewußtseins aus, weit hinaus über das Wanderer- oder Schreibertum. Schon als Kind wußte er von jedem Obstbaum der Gegend, wann dort die ersten Früchte reiften, und ... Sein erstes Buch hatte zum Titel „Die Birnendiebe“. Und bis jetzt auch konnte er an keinem fremden Obstgarten vorbeigehen ohne zumindest einen Versuch, und sei es auch bloß in Gedanken, dort zu räubern. (MN, 478)

Der Protagonist identifizierte sich mit dem Schreiben schon als Kind und verlangte bei seinen kreativen Schreibarbeiten unbedingt Ruhe. Im Schreiben geht es um die Öffnung eines Raumes, in dem das schreibende Subjekt unablässig verschwindet (vgl. Foucault 2003: 239). Als Erwachsener wird er vom ständigen Schuldgefühl für die Spaltung der Familie verantwortlich zu sein, begleitet:

Im Türrahmen - so dick waren die Mauer, daß der Bruder breitbeinig dastand bekam er von diesem noch zu hören, wie die ganze Familie unten sich gefürchtet hatte, wenn er, damals noch ein Jugendlicher, sich oben in seinem Zimmer an seinem ersten Schreiben versucht hatte. Keinen Mucks hatten sie machen dürfen. Hustete bloß einer von ihnen, oder schrammte ein Stuhl, so brüllte er schon um Ruhe. [...] Selbst, wenn er, auch das kam, selten genug, vor, lächelte und, als wäre nichts gewesen, als hätte er nicht gerade noch zu ihren Häupten getobt, ihnen übergangslos das Geschriebene vorlas, sie zum zuhören zwang - allein die Mutter brauchte nicht gezwungen zu werden -, war er ihnen unheimlich, zumal auch sein Lächeln ein eher bedrohliches war, ein Grinsen, ein schurkisches, wie nach vollbrachter Tat. (MN, 496f.)

Das Individuum wird durch mehrere kulturelle Muster geprägt. Der Ex-Autor leidet auch unter einer Schreibkrise und sucht in der Umgebung nach Inspiration für sein inneres Drängen. Welchen Einfluss die Reisen auf den Protagonisten gehabt haben, lässt sich aus folgendem Zitat erkennen:

Und zuletzt kurz vor der Abfahrt - unversehens, wie es so oft die Begebenheiten bei uns auf dem Balkan, ging es los -, geriet er mir nicht in den Blick, wie er von seinem Platz aufsprang und alle seine Gewandtaschen, ich erriet 
das, mechanisch, nach eingefleischter Gewohnheit, nach Papier und Bleistift absuchte - offenbar ohne Erfolg. Ja, wollte er denn doch wieder etwas aufschreiben? Hatte er denn vergessen, daß er einen Hautausschlag bekam, wenn er ein Blatt Papier, vor allem ein leeres, berührte, und sogar schon bei einem bloßen Papierknistern? Daß er sämtliche Bleistifte an Bord zerbrochen und in den Fluß geschmissen hatte? (MN, 60f.)

„Im Individuum gibt es ein ,tiefes’ Drängen, eine ,schöpferische' Kraft, ein ,Projekt', der Ursprungsort des Schreibens" (Foucault 2003: 248). Daraus wird deutlich, dass das „Schreibertum“ immer ein Teil der Existenz des Protagonisten bleiben wird.

\section{KULTURSPEZIFISCHE AUSPRÄGUNGEN DES FREMDEN}

„Fremdheit, die durch die Art ihres Zugangs bestimmt ist und nicht vorweg ein gesichertes Terrain des Eigenen, eine ,Eigenheitssphäre' voraussetzt, begegnet uns nicht nur in Anderen, sie beginnt im eigenen Haus als Fremdheit meiner selbst oder als Fremdheit unserer selbst" (Waldenfels 2013: 27). Der Protagonist wird sich seiner eigenen Fremdheit mit Sinneseindrücken bewusst:

Und selbst ein Geräusch, aus dem jeder sonst etwas Offenes, Freundliches, dem Sprecher rückhaltlos Gewogenes herausgehört hätte, einen Ton, den Tod der selbstlosen Erwartung, ja, einen Einklang vor vorneherein, kappte ihm auf der Stelle die Atemwege, materialisiert sich als ein Fremdkörper in seiner Luftröhre? (MN, 29)

„Das Subjekt konstituiert sich durch den Ort des Anderen, was sowohl bedeutet, dass das Objekt der Identifikation ambivalent ist, als auch, dass der aktive Vorgang der Identifikation nie rein oder holistisch ist, sondern immer aus einem Prozess der Substitution, De-placierung oder Projektion besteht" (Bhabha 2000: 241). Einerseits strebt der Ex-Autor nach der Fremdheit und Unauffälligkeit, andererseits wünscht er sich von dem sozialen Umfeld wahrgenommen zu werden:

Ihm war, als sei er über die Zeit hinaus von niemandem, von keinem lebenden Menschen wenigstens, gesehen worden. Es gab keine Erinnerung mehr an ein menschliches Auge, das ihm mit einem auch bloß flüchtigen Blick betrachtet hätte, und solch ein Wahrgenommenwerden fing an, ihm zu fehlen. [...] Etwas Sonderbares kam dazu: Er, dem es in seiner Epoche als öffentliche Person eine Pein gewesen war, auf der Straße oder wo erkannt zu werden, wünschte sich jetzt, jemand in dem Zug möge ihn endlich erkennen, ein einziger, ein einziges 
Wesen, und zwar als den, der das und das hervorgebracht hatte, auch wenn das schon nun länger her war. (MN, 376)

Das Fremdwerden kommt zum Ausdruck durch eine Wir-Gruppe, indem man die Anderen als Fremde erfährt oder indem man sich selbst Anderen gegenüber als Fremder fühlt (vgl. Waldenfels 2013: 38). Das folgende Zitat erläutert das Fremdheitsgefühl auf anschauliche Weise:

Jedenfalls äußerte sich das Zeitkranksein [...] so suggerierte die Zeitkrankheit, ausgebrochen in der Übereilung, daß das Ziel nicht nur näher, vielmehr, Herzschlag um Herzschlag, weiter wegrückte, und vor allem, daß es kein Ziel war, nicht wert dieses Namens, und daß die Menschen und die Landschaften, die einen dort etwa erwarteten, selbst die herzallerliebsten, fremd waren in dem Sinn, daß sie gar nichts bedeuteten - daß das Ziel selber in solchem Sinn die Fremde war. (MN, 313)

Mit dem Begriff „Stara Vas“ (MN, 462) ist Peter Handkes Heimatdorf Altenmarkt bei Griffen gemeint, dass in dem literarischen Werk als ein Zwischenraum interpretiert werden kann. Als der Autor sein Heimatdorf in Kärnten besucht, fühlt er sich in der bekannten Umgebung als Fremder. „Als der ,potenziell Wandernde' bildet der Fremde zwar ein ,Element der Gruppe', aber ein Element besonderer Art, das ein ,Außerhalb und Gegenüber' einschließt““ (Waldenfels 2013: 39). Bei der Beobachtung der Umgebung zeigt sich die wahrnehmbare Ausweitung der islamischen Religion:

Die arabischen Aufschriften an den Geschäften - die lateinische Schrift, hier und da, ziemlich in der Minderheit -, das Minarett und die Kopftuchfrauen, die er zuerst für Nonnen, aus dem nahen Kloster, gehalten hatte, auch die paar Verschleierten, waren da nicht das Bestimmende, eher eine Begleiterscheinung. (MN, 455)

„Die ,Zwischenräume der Kulturen’ erweisen sich als ein Gefüge weltweiter kultureller Verflechtungen, die keineswegs nur durch einen ,gleichursprünglichen' Wechseltausch geprägt sind, sondern nicht zuletzt auch durch kulturelle Differenzen, Konflikte, Hierarchien sowie durch Ungleichheiten zwischen Zentren und Peripherien“" (Bachmann-Medick 2003: 445). Nach Bhabha (2012: 29) bestehen die Zwischenräume aus „,vergangener und künftiger Zeit“. Der ehemalige Apfelkeller des Bruders wurde zu einer Versammlungsstätte, der auch die Funktion eines Zufluchtsortes zugeschrieben wird, ungebaut. In dem literarischen Werk werden auch kritische Hybridität und politische Brisanz betont:

Und nun diente es wieder als Gotteshaus, allerdings auch eher insgeheim, nicht offiziell jedenfalls, nirgends angezeigt, eine Art Krypta, oder Katakombe. 
Manche Fernfahrer - es gab auch solche - stiegen aus der Gaststube oben zu ihr hinab als zu ihrer Autobahnkirche; manche Alteingesessenen des Dorfs - es gab noch welche, und sie waren des Bruders Stammgäste - psalmodierten an den Feierabenden [...], da unter den Rosenkranz und die Marien- und Allerheiligenlitanei; und die Neuzugezogenen aus „Samarkand“ benutzten, anfangs wenige, inzwischen mehr und mehr (die der ihnen gar zu sichtbar und großmächtig gewordenen Moschee allmählich überdrüssig geworden waren), den Keller für ihr gemeinsames Freitagsgebet, bei dem die sich in dem kleinen Saal, anders als in der Moschee, leicht so eng zusammenstellen konnten, daß zwischen ihnen, wie von ihrer Religion gefordert, kein Raum blieb für das Eindringen des Schaitan, oder des bösen Dämons. (MN, 494f.)

Das Fremde wird wahrnehmbar als etwas, das durch die verschiedenen Ordnungen ausgeschlossen und eben damit hervorgebracht wird als Außerordentliches, als zugleich Prä- und Transkulturelles, das die Kulturen unterwandert und übersteigt (vgl. Waldenfels 2013: 141). In dem literarischen Text wird auch die „strikte“ Differenzierung von Kulturen betont. Diese wird deutlich als der Protagonist eine fiktive Doline im Karst oberhalb von Triest besucht:

Nicht allein dort unten, in der ausgedehnten Erdschüssel - im ganzen Karst und seinem Umland war alles Balkanische oder auch nur von ferne daran Erinnernde fern, von den Speisen über die Kleidung bis zur Musik (die besonders, nur mitteleuropäische Weisen und Instrumente hatten zu erklingen, am besten Wiener Walzer, und die Radiostationen von Mitteldorf zu Mitteldorf gaben tagtäglich den Ton vor). In der „Mitteldoline“, wie die Delana Dolina nun offiziell hie $\beta$, herrschte die Mitteleuroparegelung jedoch besonders strikt. Undenkbar da das Erschallen einer Balkanklarinette oder -trompete, das Braten eines Lamms am Spieß (von einem Spanferkel zu schweigen), das Verzehren von rohen Zwiebeln. (MN, 513)

\section{DIE HEIMAT IN DER FREMDE}

Die wirkliche Heimat kann außerhalb der ursprünglichen Heimat gefunden werden, was auch das lateinische Sprichwort Ubi bene, ibi patria besagt (vgl. Welsch 2009). Bei der Fremdheit handelt es sich um Aspekte des Ortes, des Besitzes und der Art, die dem Fremden im Gegensatz zum Eigenen sein wechselndes Gepräge geben. Das Entscheidendste dabei ist das leibhaftige Hier bzw. der Ortsaspekt, denn Fremdes verbindet sich mit der Form des Anderswo (vgl. Waldenfels 2013: 142). Der Ex-Autor wird in seiner kulturellen Formation durch mehrere kulturelle Verbindungen und Orte bestimmt: „Die Orte 
seiner Vergangenheit hatten sich verbunden mit seinem Fleisch und Blut. Keine Körperstelle an ihm, zu der nicht ein Ort gehörte. Keine Zelle so seine Überzeugung, die nicht einen Ortsnamen bereithielt" (MN, 492).

Der Ex-Autor versucht sich anhand der Imagination zu verorten, indem er eine Enklave an einem beliebten Ort bildet. Mit der Enklave erschafft er auch eine Interdependenz in der Gesellschaft. Bei der fiktiven Enklavenbindung wird das Fremde an das Eigene angeschlossen. Der Protagonist verortet sich im Pendeln zwischen seiner früheren Sich- und Erlebensweisen und den neuen, selbstkonstituierenden Wahrnehmungsweisen. Er erfährt den neuen kulturellen Raum als Freiraum. Das Schiff auf dem Fluss Morawa fungiert als Wohnort und Heimatort zugleich. Die Morawa kann auch als sinnstiftende Leerstelle und Daseinsmetapher gedeutet werden. Eine zerbrechliche Verortung ${ }^{7}$ wird auf dem Schiff gekennzeichnet: „Das Boot war nicht verankert, sondern bloß so an Bäumen oder Strommasten vertäut, und zwar derart, daß die Taue leicht und schnell zu lösen wären - eben zur Flucht, oder auch nur zum Mir-nichts-dir-nichts-Weiterfahren oder Wenden, flußauf oder flußab“ (MN, 8).

„Der Wandernde wohnt an einem Ort, doch wohnt er dort nicht mit Leib und Seele. Seine Position, die nicht einfach eine ,Gliedstellung' in der Gruppe, sondern einen gleichzeitigen Abstand zur Gruppe markiert, verschafft ihm die Möglichkeit, das Gruppengefüge mitsamt der ihm innewohnenden Fremdheit als solches zu erfassen" (Waldenfels 2013: 39). Der Ex-Autor wird sich seiner Individualität bewusst, nichtsdestotrotz möchte er die Menschheit in seiner Nähe spüren: „Wenn es ihn zu Menschen zog, dann zu vielen, und dort, wo die sich zusammenballten, anonym, also weniger in die Dörfer und die Kleinstädte, aber in der Regel genügte es, daß er sie dann nicht allzu fern fühlte " (MN, 332).

Der Protagonist versucht auch alle seine Beziehungen und emotionalen Bindungen auf Distanz zu halten: „Die Liebe, insbesondere die zu einer Frau, gehört nicht zu meinem Wortschatz, auch nicht Heimkehr und Heimat " (MN, 370). Trotz seiner ethnischen österreichischen Herkunft identifiziert sich das Individuum mit dem Balkan:

Weniger nach Österreich zog es ihn als von dort weiter, nachhause, ja, nachhause auf den Balkan, auf das Boot an der Morawa. Er fühlte? dachte? Nein, er wußte sich mit dem Fluß und der Enklave von Porodin verbunden; wußte sich dem Ort verpflichtet, auch wenn kaum jemand dort sich um ihn scherte, geschweige denn ihn nötig hätte. (MN, 306)

Das Boot auf der Morawa ist schon mehrere Jahre seine „kleine Heimat“ (MN, 47) und „Domovina“ (MN, 47) zugleich. Welsch (2009) argumentiert, „,ie erste Heimat sei in gewissem Sinn nur als zweite Heimat wirkliche Heimat, erst dann nämlich, wenn man sich (angesichts auch anderer Möglichkeiten) bewusst zu ihr entschieden, sie nachträglich

7 Die Verortung bezieht sich auf grenzüberschreitende Wanderungsbewegungen und auf multiple Stimmen, die im literarischen Werk auftreten. 
eigens gewählt und bejaht habe. Nur dann sei ,Heimat' keine naturwüchsige, sondern eine kulturelle und humane Kategorie." In der Erzählung hat der Fluss eine symbolische Bedeutung der Vergänglichkeit:

Nicht einmal ein Fluß, nicht einmal die Morawa vor den Fenstern, die ganz und gar keine Bullaugen waren. Auffällig das grelle Licht in den Ritzen der Schwingtür mit der Aufschrift „KUHINJA“, Küche. Die Tür aufgestoßen - und wieder nichts, wieder die Leere. Geblendetes Stehen in der Sonne. Keine Spur von einem Schiff, von der MORAWISCHEN NACHT? Was gerade noch ein Schiff gewesen war, schrumpfte zum Einbaum, und der Einbaum sank. Und der Fluß, die Morawa? Die Morawa versiegelte. Und Porodin war doch keine Enklave, nie eine gewesen. Die balkanischen Enklaven lagen woanders. (MN, 556)

Die Morawa kann als Quelle der schöpferischen Tätigkeit und als Sinnbild des Unbewussten interpretiert werden. Das anregende Klima, das die Sinne aktiviert und die Freiheit, die sich mit dem Fluss verbindet, sorgen dafür, dass sich der Protagonist bzw. der „Ex-Autor“ als präsenter Autor wiederfindet, sich seiner Existenz bewusst wird und ein neues Buch in einer morawischen Nacht auf dem Boot verfasst. Der Autor wird sich seiner Handlungen kritisch bewusst: „Ein Irrtum, das ging ihm jetzt auf, war dann seine Suche nach den weiten Horizonten gewesen. Ein Irrtum? Eine Verirrung. " (MN, 58)

„Die Alternative von Hiersein oder Dortsein, die sich darauf beschränkt Eigenorte zu vervielfältigen, wird überwunden in einer Ortsverschiebung, die im Aufschub, in der Verzögerung einen zeitlichen, genauer gesagt einen diachronen und heterochronen Charakter annimmt." (Waldenfels 2013: 203). In den relationalen Kategorien des Fremden und des Eigenen führt das Selbstverstehen zum Fremdverstehen und jeder, der seine Erfahrungen und seine angestammte Welt mit fremden Augen ansieht, kann in keiner der beiden Welten mehr naiv zu Hause sein (vgl. Wierlacher 1983: 10).

\section{9}

\section{SCHLUSSFOLGERUNG}

Handke bezieht sich in seiner Erzählung auf vernachlässigte Orte kultureller Produktion, die als Zwischenräume gedeutet werden können. Er konstruiert kulturelle Prozesse der Verfremdung, des Austauschens sowie des Konflikts und thematisiert die hybride Ambivalenz. Diese wird anhand von Handlungen und Interventionen des Protagonisten veranschaulicht. Nach Welsch (2009) „,wirkt sich die zeitgenössische kulturelle Durchdringung - die Transkulturalisierung- auch auf Grundfragen des individuellen und gesellschaftlichen Selbstverständnisses aus." Die vorkommenden fiktiven und realen Orte verbinden sich mit historisch-sozialen Handlungszusammenhängen in einem Prozess der kulturellen Codierung. Die fiktiven Orte erschaffen jenen Erzählraum und 
jene Erzählhaltung, in denen sich die Leser erst einmal ihren eigenen Ort schaffen müssen, der meistens gleich ein Nicht-Ort ist, unbestimmt, abseits gelegen von jenen Orten und Zeiten, die auf den Landkarten und in den Geschichtsbüchern verzeichnet sind (vgl. Fetz 2009: 199). Die Hauptfigur der Erzählung bewegt sich auf der Ebene interkultureller Beziehungen in Veränderungsspielräumen von Zwischenpositionen, in denen sie sich ihrer Existenz erst bewusst wird. Die Zwischenräume als Spannungsfeld spielen eine große Rolle bei der Identitätsfindung und Etablierung der Beziehungsverhältnisse zwischen den Kulturen. Wierlacher (2003: 262) drückt diese Tatsache folgendermaßen aus: „Diese zwischenkulturelle Mitte macht nicht ortlos, und sie nimmt niemandem die Selbstgewissheit angestammter oder entworfener Zugehörigkeit, sondern stiftet als neue und zusätzliche Seinsqualität ein Stück Lebensqualität und Zugehörigkeit.“ Mit diesem literarischen Text kann die postkoloniale Wende mit Bezug auf europäische Traditionen positioniert werden. Mit der anschaulichen Lebensweise des Protagonisten entwickelt sich ein Konzept des Individualismus, der sich mit Menschenrechten und Moralität auseinandersetzt. Auch Phänomene des menschlichen Lebens und das kulturelle Verhältnis zu Zeit, Tod, Raum, Gott, Liebe, Natur und Ähnlichem kommen zum Ausdruck. Eine Frage bleibt bei Handke allerdings offen, und zwar, wo soll man die Grenzen zwischen Sprachen und Kulturen ziehen? Eines steht fest, „[d]er Autor ist derjenige, der es möglich macht, sowohl die Präsenz bestimmter Ereignisse in einem Werk wie auch deren Transformation, deren Deformationen, deren verschiedene Modifikationen zu erklären“ (Foucault 2003: 246).

\section{LITERATURVERZEICHNIS}

BACHMANN-MEDICK, Doris (1999) $1+1=3$ ? Interkulturelle Beziehungen als »dritter Raum«. In: Weimarer Beiträge. Zeitschrift für Literaturwissenschaft 4 (45. Jahrgang), 518-531.

BACHMANN-MEDICK, Doris (2003) Kulturanthropologische Horizonte interkultureller Literaturwisseschaft. In: Handbuch interkulturelle Germanistik, hrsg. von Alois Wierlacher und Andrea Bogner, Stuttgart/Weimar: J. B. Metzler Verlag, 439-448.

BACHMNANN-MEDICK, Doris (2014) Cultural Turns. Neuorientierungen in den Kulturwissenschaften, 5. Auflage mit neuem Nachwort, Reinbek bei Hamburg: Rowolth Taschenbuch Verlag.

BHABHA, Homi K. (2000) Die Verortung der Kultur. Mit einem Vorwort von Elisabeth Bronfen. Deutsche Übersetzung von Michael Schiffmann u. Jürgen Freudl, Tübingen: Stauffenburg Verlag.

BHABHA, Homi K. (2012) Über kulturelle Hybridität. Tradition und Übersetzung. Aus dem Englischen von Kathrina Menke, hrsg. und eingeleitet von Anna Babka und Gerald Posselt, Wien/Berlin: Verlag Turia + Kant. 
DEICHMANN, Thomas (2009) Literatur und Reisen mit Peter Handke. In: Peter Handke. Freiheit des Schreibens - Ordnung der Schrift, hrsg. von Klaus Kastberger, 1. Auflage, Wien: Paul Zsolnay Verlag (= Profile. Magazin des Österreichischen Literaturarchivs der Österreichischen Nationalbibliothek, Band 16), 183-193, auch online verfügbar auf Handkeonline. 1. September 2016. http://handkeonline.onb.ac.at/forschung/pdf/ deichmann-2009.pdf/.

FETZ, Bernhard (2009) Peter Handkes balkanische „Geographie der Träume“: Die Morawische Nacht. In: Peter Handke. Freiheit des Schreibens - Ordnung der Schrift, hrsg. von Klaus Kastberger, 1. Auflage, Wien: Paul Zsolnay Verlag (= Profile. Magazin des Österreichischen Literaturarchivs der Österreichischen Nationalbibliothek, Band 16), 194-204, auch online verfügbar auf Handkeonline. 1. September 2016. http://handkeonline.onb.ac.at/forschung/pdf/fetz-2009.pdf/.

FOUCAULT, Michel (2003) Schriften zur Literatur, hg. von Daniel Defert und François Ewald unter Mitarbeit von Jacques Lagrange. Aus dem Französischen von Michael Bischoff, Hans-Dieter Gondek und Herrmann Kocyba, Frankfurt am Main: Suhrkamp Verlag.

GUTJAHR, Ortrud (2002) Interkulturalität. Neuere deutsche Literatur. In: Germanistik als Kulturwissenschaft. Eine Einführung in neue Teoriekonzepte, hrsg. von Claudia Benthien und Hans Rudolf Velten, 1. Auflage, Reinbek bei Hamburg: Rowohlt Taschenbuch Verlag, 345-369.

HANDKE, Peter (2009) Die morawische Nacht. Erzählung, Frankfurt am Main: Suhrkamp Taschenbuch Verlag.

HERWIG, Malte (2012) Meister der Dämmerung. Peter Handke. Eine Biographie, Pantheon-Ausgabe, München: Pantheon Verlag.

LESKOVEC, Andrea (2011) Einführung in die interkulturelle Literaturwissenschaft. Darmstadt: Wissenschaftliche Buchgesellschaft.

TEXT UND BÜHNE (2013) „Peter Handke im ZDF Nachtstudio Gespräch mit Volker Panzer am 10.3.2008“. 2. September 2016. https://www.youtube.com/ watch?v=y8PM4YMEXhQ/.

WALDENFELS, Bernhard (2013) Topographie des Fremden. Studien zur Phänomenologie des Fremden 1, 6. Auflage, Frankfurt am Main: Suhrkamp Taschenbuch Verlag.

WELSCH, Wolfgang (2009) Was ist eigentlich Transkulturalität? In: Hochschule als transkultureller Raum? Beiträge zu Kultur, Bildung und Differenz, hrsg. von Lucyna Darowska und Claudia Machold, Bielefeld: Transcript Verlag.

WIERLACHER, Alois (1983) Mit fremden Augen. Vorbereitende Bemerkungen zu einer interkulturellen Hermeneutik deutscher Literatur. In: Jahrbuch Deutsch als Fremdsprache 9, 1-16.

WIERLACHER, Alois (2003) Interkulturalität. In: Handbuch interkulturelle Germanistik, hrsg. von Alois Wierlacher und Andrea Bogner, Stuttgart/Weimar: J. B. Metzler Verlag, 257-264. 


\section{POVZETEK}

\section{Konstrukcija kulturne tujosti v pripovedi Moravska noč Petra Handkeja}

Članek poskuša na podlagi pripovedi Moravska noč pisatelja Petra Handkeja prikazati različne kulturne pojave znotraj socialnega sistema v obliki relacijskih pojmov lastnega in tujega. Poudarek je na iskanju lastne identitete posameznika v kulturno pluralnem prostoru Evrope. Avtor, ki se poistoveti s protagonistom, eksistencialno pripoveduje o kulturni razsežnosti lastnega izkustva $\mathrm{v}$ medkulturnem dialogu. $Z$ vidika tujosti in drugačnosti se giblje med kulturami in zaznava kulturne vzorce hibridnosti. Razcepljenost lastnega jaza je ponazorjena z estetskim doživljanjem odtujenosti in dilemo med lastnim in tujim. Analiza filozofskih misli, vedenjskih vzorcev ter medosebne komunikacije v literarnem delu omogoča razumevanje tujosti, ki je ključnega pomena pri zavedanju lastne pripadnosti v kulturnem procesu.

Ključne besede: fenomenologija tujosti, medkulturnost, vprašanje identitete, kulturna izkušnja, literarni diskurz

\section{ABSTRACT}

\section{Construction of the Cultural Alien in The Morawian Night, a Story by Peter Handke}

The article, based on the story The Morawian Night by Peter Handke, attempts to explain divergent cultural phenomena within the social system in the form of relational concepts of "selfhood" and "otherness". The focus is on the search of an individual's own identity in the multicultural setting of Europe. The author, who identifies himself with the protagonist, speaks in existential terms about the cultural dimension of his own experience in intercultural dialogue. With the awareness of otherness and foreignness, he moves among cultures and perceives the cultural dimensions of hybridity. The division of one's self is exemplified with aesthetic experiencing of alienation, and the dilemma between what is one's own and what is foreign. The analysis of philosophical thoughts, behaviour samples, and interpersonal communication make it possible to understand foreignness, which has a crucial role in understanding one's belonging in the cultural process.

Keywords: Phenomenology of the Alien, interculturality, the question of identity, cultural experience, literary discourse 